This is the author's version of a work that was published in the following source:

Braun, M.; Dengiz, T.; Mauser, I.; Schmeck, H. (2016).

Comparison of Multi-objective Evolutionary Optimization in Smart Building Scenarios 므.

Applications of Evolutionary Computation (EvoApplications): 19th European Conference, Proceedings Part 1, Porto, Portugal, 30th March - 1st April 2016. Ed.: G. Squillero, 443458, Springer, Berlin.

https://doi.org/10.1007/978-3-319-31204-0_29

Please note: Copyright is owned by the author(s) and / or the publisher. The commercial use of this copy is not allowed. 


\title{
Comparison of Multi-objective Evolutionary Optimization in Smart Building Scenarios
}

\author{
Marlon Braun ${ }^{1}$, Thomas Dengiz ${ }^{1}$, Ingo Mauser ${ }^{2}$, and Hartmut Schmeck ${ }^{1,2}$ \\ 1 Karlsruhe Institute of Technology - Institute AIFB, 76128 Karlsruhe, Germany \\ \{marlon.braun, schmeck\}@kit.edu, \\ thomas.dengizestudent.kit.edu \\ 2 FZI Research Center for Information Technology, 76131 Karlsruhe, Germany \\ mauserefzi.de
}

\begin{abstract}
The optimization of operating times and operation modes of devices and systems that consume or generate electricity in buildings by building energy management systems promises to alleviate problems arising in today's electricity grids. Conflicting objectives may have to be pursued in this context, giving rise to a multi-objective optimization problem. This paper presents the optimization of appliances as well as heating and air-conditioning devices in two distinct settings of smart buildings, a residential and a commercial building, with respect to the minimization of energy costs, $\mathrm{CO}_{2}$ emissions, discomfort, and technical wearout. We propose new encodings for appliances that are based on a combined categorization of devices respecting both, the optimization of operating times as well as operation modes, e.g., of hybrid devices. To identify an evolutionary algorithm that promises to lead to good optimization results of the devices in our real-world lab environments, we compare four state-of-the-art algorithms in realistic simulations: ESPEA, NSGA-II, NSGA-III, and SPEA2. The results show that ESPEA and NSGA-II significantly outperform the other two algorithms in our scenario.
\end{abstract}

Keywords: Energy management system $\cdot$ Smart building $\cdot$ Evolution-ary algorithm · Multi-objective optimization

\section{Introduction}

In the face of a potential climate change that may be induced by man-made carbon dioxides, many countries started to change their power generation from fossil to renewable energy sources (RES). Coal-fired power plants are being replaced by plants emitting less carbon dioxide, such as wind turbines and photovoltaic (PV) systems that exploit solar radiation. Usually, their generation is intermittent and hardly controllable. This is already leading to high production peaks causing voltage problems and overloads in distribution grids as well as to periods with barely any generation by RES, meaning that almost all the needed power still has to be produced by conventional power generation [1]. 
One way to deal with this problem of intermittent generation is to build additional energy storage systems that are able to balance the fluctuations out. Unfortunately, this is quite expensive, as for instance batteries, and often leads to public resistance, e. g., in case of pumped hydro storage plants. Another way would be a change in the central paradigm of power generation and consumption. At any time, the generation in the electricity grid has to be in balance with the consumption to keep the system stable. Currently, generation follows consumption in the energy system. This may change to consumption follows production by using measures of demand side management (DSM). DSM targets on the flexibilization of the traditional demand side of the power grid, which is nowadays also generating an increasing share of power, in particular by RES, and on increasing the self-consumption as well as selfsufficiency of local energy systems [2].

We consider the optimization of two different settings of smart buildings in a multi-objective context: one scenario consists of a smart residential building (SRB), the other scenario of a smart commercial building (SCB). In both scenarios, the operation times and operation modes of household appliances as well as heating, ventilation, and air-conditioning (HVAC) devices are optimized with respect to the minimization of energy costs, $\mathrm{CO}_{2}$ emissions, and technical wearout as well as to the maximization of comfort, i.e., minimization of dis-comfort. To optimize the appliances and devices, we propose novel encodings for appliances that have advantages over existing encodings, which have been used so far in the community. These encodings are based on a combined categorization of the appliances and devices that respects both, the optimization of operation times as well as the optimization of operation modes, e.g., of hybrid appliances. We compare four multi-objective evolutionary algorithms (MO-EA) on these scenarios-ESPEA [3], NSGA-II [4], NSGA-III [5], and SPEA2 [6] - that have either been applied successfully in past studies or appear to be suitable candidates for optimizing the scenarios found in smart buildings.

Section 2 provides an overview of similar approaches to multi-objective optimization in energy management systems. In Sect. 3, the general approach, the modeling of devices, and the encodings of the devices in evolutionary algorithms are outlined. Section 4 presents the scenarios and setups that have been chosen to evaluate different algorithms for multi-objective optimization. The simulation results are analyzed and discussed in Sect. 5. We summarize and conclude the paper in the final Sect. 6 and provide an outlook on further research.

\section{Related Work}

There are numerous building energy management systems (BEMS) that have been used in simulations and in real-world environments. Usually, they are based on some kind of categorization of the devices to generalize and ease their optimization by deriving appropriate device representations. In the following sub-sections, we first present different device categorizations, before giving an overview of approaches to optimization in BEMS and showing some typical device representations. 


\subsection{Categorization of Devices in Buildings}

In the literature, there is a multitude of different device categorizations. For instance, Kok et al. [7] propose device categories that focus on the dimensions of when is it or may it be operated, e.g., stochastically or based on user interaction, and what is the constraint of operation, e.g., limiting buffer or storage.

In contrast, $\mathrm{Ha}$ et al. [8] propose a categorization that focuses on the services that are provided by the devices. The first dimension is similar to the previous categorization and targets on time and availability (permanent services versus temporary or timed services). The second dimension distinguishes whether the service is provided directly to the user or whether it is an ancillary service only (enduser services versus support services). The third dimension addresses the modifiability of the service by the BEMS (modifiable versus non-modifiable).

Nestle et al. [9] add the differentiation whether it is load or generation, whereas Soares et al. [10] emphasize the potentials of control and modifiability by the BEMS by distinguishing uncontrollable, reparameterizable, interruptible, and shiftable loads. Mauser et al. [11] separate modifiability into two dimensions. The first dimension, which is called temporal degree of freedom, defines whether a device is deferrable or interruptible. The second dimension is called energy-related degree of freedom and defines whether a device has alternative profiles or is multivalent/hybrid, i.e., supports the utilization of different energy carriers.

\subsection{Heuristic and Multi-objective Optimization in Building Energy Management Systems}

Energy management systems have to optimize the energy consumption and generation iteratively to adapt them continuously to changing conditions and states. This process of reoccurring optimization in BEMS shall be executed on computers that have a low energy consumption and thus small memory space and low computation power. Therefore, heuristics promise to achieve this task of optimization efficiently and in particular evolutionary algorithms have been used quite often, for instance in [11-15]. Other approaches also use other meta-heuristics, e.g., particle swarm optimization [16, 17], to optimize the configuration [12] or the scheduling [11, 13-17] of devices.

Usually, the optimization is done with respect to a single objective, which most of the time is the total costs. Nevertheless, there are also approaches taking other objectives into account that are not directly related to costs. For instance, De Oliveira et al. [18], who use a direct solver, consider economic costs and user comfort. Soares et al. [14] use a MO-EA, more precisely the genetic algorithm NSGA-II [4], to optimize the operation times of devices in a residential building with respect to energy costs and user satisfaction. Salinas et al. [19] develop their own MO-EA, which they call Load Scheduling With an (o-Approximate) Evolutionary Algorithm. They optimize the device operation in multiple buildings with respect to the minimization of the total energy consumption costs and the maximization of usefulness, i.e., gross income of the overall community and comfort of individuals that living in it. They show that an approach that enforces diversity in the population leads to better results within shorter time. 


\subsection{Representation of Devices}

Allerding et al. [13] use a matrix of binary values determining the starting times of the devices as the representation of the optimization problem. Therefore, at minute resolution, the next $24 \mathrm{~h}$ are discretized to 1440 slots and every col-umn represents one minute. A similar approach has also been used by Zhao et al. [15], where the operation starting times of appliances and HVAC devices are optimized using a vector of binary strings that encodes the slot of the day, when the appliance with its fixed operation duration will be started.

Mauser et al. [20] propose a more distinct approach to the encoding of the operating times of the devices in the genotypes: the overall optimization problem is represented by a bit string. Every device uses a dedicated part of the bit string for the individual encoding. The starting times of appliances are encoded as rel-ative times within the time window of optimization. Interruptible devices and devices with alternative load profiles, such as hybrid devices, are also supported by adding more bits to the bit string that determine the length of interrup-tions or enumerate the alternatives, respectively. The operating times of a micro combined heat and power plant (micro-CHP) are encoded as transitions of an automaton.

Soares et al. [14] encode the starting times of the appliances in a string of integers, i.e., the overall problem is represented by a vector of integer values. The optimization horizon is set to the next $36 \mathrm{~h}$ and discretized to time slots of one minute. This approach is similar to Salinas et al. [19], who use a representation that uses a vector of real values.

\section{Approach and Modeling of Devices}

This section outlines the general approach, the categories for the devices used in this paper, the encodings of the devices in evolutionary algorithms, and the objective functions used in the optimization.

\subsection{General Approach}

The general approach of this paper to the optimization of devices in smart buildings is as follows: Firstly, we define several device categories and assign devices that are typically found in buildings to these categories. Then, we use a dedicated generic encoding for each category that fits the optimization of such devices. Finally, we distinguish several objectives that are usually conflicting and thus leading to a multi-objective optimization problem.

\subsection{Device Categories}

The device categories used in this paper are based on the categorizations in [11, 20] and can be found in Table 1. Firstly, we identify whether the device is optimizable. Secondly, we distinguish whether an appliance has load-flexibility, 
Table 1. Proposed device categories.

\begin{tabular}{|c|c|c|c|}
\hline \multicolumn{4}{|l|}{ Category } \\
\hline Load-flexibility & Interruptibility & Description & Example \\
\hline \multicolumn{2}{|l|}{ Non-optimizable } & $\begin{array}{l}\text { Device may only be con- } \\
\text { trolled by the user }\end{array}$ & Hob, oven; PV system \\
\hline None & (Yes) & $\begin{array}{l}\text { Starting time of the } \\
\text { device may be opti- } \\
\text { mized within a defined } \\
\text { period }\end{array}$ & $\begin{array}{l}\text { Traditional dishwasher, } \\
\text { tumble dryer, washing } \\
\text { machine }\end{array}$ \\
\hline None & Yes & $\begin{array}{l}\text { Operation cycle of } \\
\text { device may be split into } \\
\text { one or more phases that } \\
\text { are separated by pauses }\end{array}$ & $\begin{array}{l}\text { Interruptible dishwasher, } \\
\text { tumble dryer, washing } \\
\text { machine; micro-CHP }\end{array}$ \\
\hline Yes & None & $\begin{array}{l}\text { Device has alternative } \\
\text { load profiles for the } \\
\text { same operation cycle }\end{array}$ & $\begin{array}{l}\text { Lighting, heat pump, } \\
\text { heating element; load- } \\
\text { flexible appliances }\end{array}$ \\
\hline Yes & Yes & $\begin{array}{l}\text { combination of the two } \\
\text { above }\end{array}$ & $\begin{array}{l}\text { Air-conditioning, gas- } \\
\text { fired boiler; interruptible } \\
\text { load-flexible appliances }\end{array}$ \\
\hline
\end{tabular}

i.e., alternative load profiles, and whether the device's operation cycle may be deferred or may be even interrupted. Deferability is simply handled as a special form of interruptibility, which is limited to a single interruption at the very beginning of a possible operation cycle.

In contrast to [11, 20], the micro-CHP, which is usually a gas-fired engine that generates electricity and hot water simultaneously, i.e., working as power generator and central heating, is modeled similarly to the appliances. The micro-CHP is a device having unlimited interruptibility but no load-flexibility, whereas the air-conditioning has not only unlimited interruptibility but also loadflexibility in form of different cooling power levels.

The flexibility of the devices is either limited to a temporal flexibility window that, in reality, would be set individually by a user per operation cycle or it is limited due to the capacity of an energy storage, such as a hot water storage tank.

\subsection{Device Modeling and Encoding}

Interruptible, load-flexible appliances form the basis of our modeling approach as all other device categories can be derived from them. For example, interruptible devices may be interpreted as interruptible, load-flexible devices that possess one operation mode only in every phase. Each device j possesses a user-provided earliest starting time, denoted by release time $r^{j}$, and a latest finishing time, called deadline $\mathrm{d}^{\mathrm{j}}$.

Interruptible devices possess multiple phases of operation. The length of an individual phase $\mathrm{i}$ is denoted by $\mathrm{p}_{\mathrm{i}}^{\mathrm{j}_{i}}$. We model interruptibility by associating 


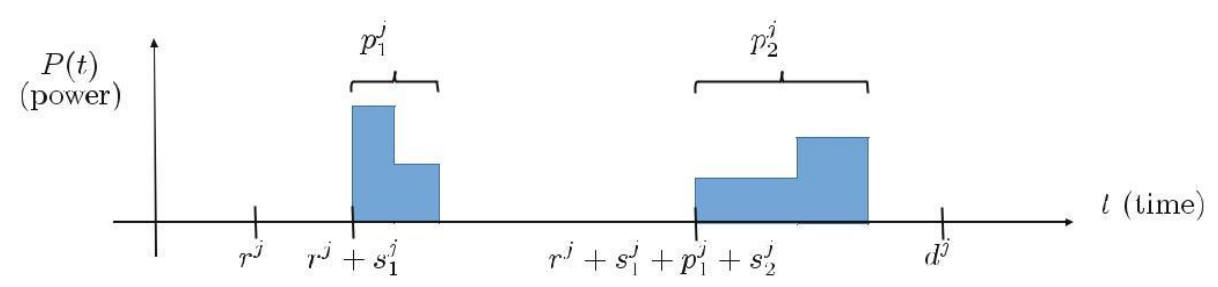

Fig. 1. Visualization of the encoding of interruptible devices.

every phase with a decision variable $s_{j}^{j}$. Each $s_{j}^{j}$ is an integer that states the time, at which phase $i$ is executed. In contrast to other approaches, every $s^{j}{ }_{i}$ uses its earliest time of execution instead of the system time as reference point. Hence, $s j_{i}$ $=0$ implies that phase $i$ is started directly after phase $i-1$. Figure 1 illustrates the approach. Constraint-handling is simplified using this encoding, since it is suffcient to check whether the last phase terminates before $d^{j}$. Different operation modes are modeled by integer encoding as well. For each phase $i$, the variable $a^{j} j_{j}$ represents the operation mode that is chosen.

HVAC devices can be started an arbitrary number of times during the optimization horizon. We incorporate this feature by introducing an integer encoded variable $n^{j}$ that states the number of possible interruptions and thus opera-tion cycles. Minimum and maximum operation as well as minimum interruption time are implemented for HVAC devices limiting the number of feasible opera-tion cycles. We apply a thermodynamical model to describe the changes in room and boiler temperatures that considers heat losses, energy conversion efficiencies, and body heat dissipation ${ }^{1}$. Finally, lighting is modeled as an interruptible device with multiple operation modes reflecting different light intensities.

Summarizing our approach, a single device is modeled using the decision variables listed below. Note that variables are omitted if they are not applicable for the given device and the sizes of $s^{j}$ and $a^{j}$ change dynamically depending on $n^{j}$ :

$$
\begin{aligned}
& s^{j}: \text { vector of starting times for each individual phase } \\
& a^{j}: \text { vector of operation modes for each phase } \\
& n^{j}: \text { number of operation cycles }
\end{aligned}
$$

\subsection{Objective Functions}

Our model considers a fixed, discretized time horizon for optimization. For the sake of clarity, most functions in this section only compute values at any time t. Selfevidently, the objective values are obtained by summing up function values across all time slots. All objective functions in our model are minimized.

\footnotetext{
${ }^{1}$ A complete description of this model goes beyond the scope of this paper, but is available on request.
} 
Energy costs are based on the electricity $\mathrm{Pb}(\mathrm{t})$ bought from the utility for a timevariable price $\mathrm{pb}(\mathrm{t})$, the gas $\mathrm{G}(\mathrm{t})$ consumed at a fixed price $\mathrm{pg}$ per unit and the exceedance of a time-variable load limitation, which is penalized using a cost function $\mathrm{S}(\mathrm{t}, \mathrm{Pb}(\mathrm{t}))$. Excess energy generated by the $\mathrm{PV}$ system and the microCHP is sold to the utility for $\mathrm{C}_{f}(\mathrm{t})$. Remuneration depends on the proportion of energy generated by the PV system and the micro-CHP, respectively.

$$
\mathrm{C}(\mathrm{t})=\mathrm{Pb}(\mathrm{t}) \cdot \mathrm{Pb}_{\mathrm{b}}(\mathrm{t})-\mathrm{C}_{f}(\mathrm{t})+\mathrm{Pg}_{\mathrm{g}} \cdot \mathrm{G}(\mathrm{t})+\mathrm{S}\left(\mathrm{t}, \mathrm{Pb}_{\mathrm{b}}(\mathrm{t})\right) \text {. }
$$

Carbon dioxide emissions are related to the electricity that is consumed from the grid and to local gas consumption. A time-variable signal $e(t)$ that depends on the current energy mix in the electricity grid determines the $\mathrm{CO}_{2}$ caused by consuming electricity from the grid. The constant eg describes the emissions per unit of gas consumed.

$$
E(t)=e(t) \cdot P b(t)+e g \cdot G(t)
$$

Thermal user discomfort occurs if the room temperature $\mathrm{T}(\mathrm{t})$ falls below a predefined lower threshold $\mathrm{T}^{\mathrm{I}}$ or exceeds an upper bound $\mathrm{T}^{\mathrm{u}}$. Otherwise, no discomfort occurs.

$$
\text { DT }(t)=T^{\prime}-T(t) \cdot 1 T(t)<T^{\prime}+\left(T(t)-T^{u}\right) \cdot 1 T(t)>T^{u} \text {. }
$$

Lighting user discomfort is modeled in the same manner as thermal discomfort. We also consider a lower bound $\mathrm{L}^{\mathrm{I}}$ and an upper illumination threshold $\mathrm{L}^{\mathrm{u}}$ . In our model, the current illumination level $\mathrm{L}(\mathrm{t})$ is the sum of daylight and artificial light.

$$
D L(t)=L^{\prime}-L(t) \cdot 1 L(t)<L^{\prime}+\left(L(t)-L^{u}\right) \cdot 1 L(t)>L^{u} .
$$

Technical wearout is modeled as the number of total interruptions and thus start-ups across all HVAC devices. Let $\mathrm{J} H$ V AC denote the set of all HVAC devices.

$$
W=\sum_{j \in H V A C} n^{j}
$$

Smart residential buildings are optimized with respect to total energy costs, carbon dioxide emissions, thermal discomfort, and technical wearout, whereas smart commercial buildings consider all five objectives, i.e., including lightingbased user discomfort.

\section{Scenarios and Simulation Setup}

The simulation has been implemented in version 4.5 of the jMetal framework [21] and its code is publicly available on Sourceforge ${ }^{2}$. All data that was used in the study is contained within the repository.

$\overline{2 \text { http://sourceforge.net/projects/jmetalbymarlonso/. }}$ 


\subsection{Scenarios}

We consider the seven scenarios in our study that are listed in Table 2. These scenarios reflect different challenges that potentially affect the eff ectiveness of the optimization eff ort. All SRBs comprise a washing machine, a tumble dryer, a dishwasher, a micro-CHP, a condensing boiler, and a PV system. Household appliances possess different operation modes as depicted in Table 3. Temporal flexibilities are defined by release times and deadlines. The SCB possesses a micro-CHP, a condensing boiler, and a lighting system.

Power consumption profiles of the appliances have been obtained from measurements in our laboratory environments. Real, existing products, which are listed in Table 4, served as blueprints for the simulated HVAC devices used in this study. Technical data was up-scaled for the commercial building scenario and missing data was amended by our own considerations.

Floor spaces and ceiling heights were set to $130 \mathrm{~m}^{2}$ and $2 \mathrm{~m}$ (SRB) and 900 $\mathrm{m}^{2}$ and $3 \mathrm{~m}$ (SCB), respectively, taking German legislation for occupational safety into consideration. Outside temperatures have been extracted from the online weather portal wetter.com at an hourly resolution. Measurements were taken in Karlsruhe (in case of the SRB) and Freiburg (CB). Solar radiation data is depicted in Fig. 2.

The German standard load profiles of households HO (SRB) and small enterprises G1 (CB) provided by the German Association of Energy and Water Industries (BDEW) served as estimates for the load of non-deferrable appli-ances. Time-variable electricity prices correspond to those employed in the project iZeus [22]. We used the same load limitation signal as employed by Allerd-ing et al. [13]. The penalty for exceedance was set as paying twice the current price. A natural gas price of 9.16 Cents per kWh was chosen, which reflect current tariffs in Germany. Feed-in tariffs are based on the German Renewable Energy Act with 12.56 Cents per kWh for PV systems and 8.53 Cents per kWh for micro-CHPs. $\mathrm{CO}_{2}$ emissions of power obtained from the grid is based on data from the Fraunhofer

Table 2. Overview of the scenarios examined.

\begin{tabular}{l|l|l|l}
\hline Scenarios & Building type & Date & Optimization horizon \\
\hline RW1 - RW3 & Smart residential building & 7 January 2015 & $00: 00-23: 59$ \\
\hline RS1 - RS3 & Smart residential building & 4 June 2015 & 00:00 - 23:59 \\
\hline CB & Smart commercial building & 13 March 2014 & 07:00 - 19:00 \\
\hline
\end{tabular}

Table 3. Household appliances flexibility.

\begin{tabular}{l|l|l|l|l}
\hline & RW1/RS1 & RW2/RS2 & RW3/RS3 & Temporal flexibility \\
\hline Washing machine & deferrable & interruptible & deferrable and load-flexible & 08:00-17:00 \\
\hline Tumble dryer & deferrable & interruptible & interruptible and load-flexible & 18:00-21:00 \\
\hline Dishwasher & deferrable & interruptible & interruptible and load-flexible & $10: 00-18: 00$ \\
\hline
\end{tabular}


Table 4. Technical data of HVAC devices and lighting.

\begin{tabular}{l|l|l}
\hline Appliance & Product & Manufacturer \\
\hline Micro-CHP & ecoPOWER 1.0 & Vaillant \\
Condensing boiler & Logamax plus GBH172 & Buderus \\
Hot water storage tank & VITOCELL 100-E & Viessmann \\
Lighting & SP482P & Philips \\
\hline \multicolumn{2}{|l}{} \\
& January _ June
\end{tabular}

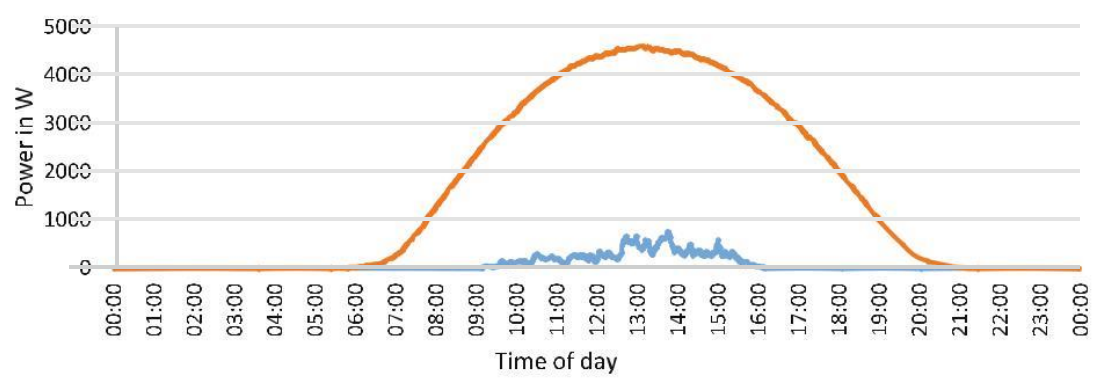

Fig. 2. Solar radiation obtained from measurements in our laboratory environments.

Institute of Solar Energy ${ }^{3}$. Hot water consumption profiles were obtained from Directive 2010/30/EU of the European Commission ${ }^{4}$ and data for computing the visual comfort was taken from Reinhart et al. [23, p. 176]. Lower and upper bounds for temperature and lighting were set to $19.5^{\circ} \mathrm{C}$ and $20.5^{\circ} \mathrm{C}$ (January), $21.5^{\circ} \mathrm{C}$ and $22.5^{\circ} \mathrm{C}$ (June), and $20.5^{\circ} \mathrm{C}$ and $21.5^{\circ} \mathrm{C}$ (March), and 750 Lux and 1000 Lux, respectively.

\subsection{Algorithms and Settings}

We chose four different state-of-the-art evolutionary algorithms for optimizing the presented scenarios. All algorithm configurations were taken from their original publications if not stated otherwise.

NSGA-Il's capability of solving problems in energy informatics has been demonstrated in several publications [14, 24], making it an ideal candidate for this study. The algorithm uses non-dominated sorting to rank its population of solutions into different tiers of non-dominated fronts for deciding which solutions prevail to the next generation. The selection mechanism is amended by the crowding distance metric if a front cannot be fully accommodated in the next generation. Crowding distance is a density measure that quantifies the volume of the smallest cuboid than can be circumscribed around a solution without enclosing any other members of the current front. 
The successor to NSGA-II is the NSGA-III algorithm that is specifically tailored to solve problems in three and higher dimensions. It uses reference points instead of crowding distance as diversity preserving mechanism. Objective values are normalized using extreme solutions and the currently best estimate of the utopia point. Reference points are projected on the hyperplane spanned by the translated extreme solutions. Solutions are associated with the reference point to which their Euclidean distance of the line between origin and reference point is minimal. Solutions are selected such that they are equally distributed over all reference points. Equidistant reference points from Deb and Jain [5] were chosen for this study.

SPEA2 has also been successfully applied in solving problems in energy informatics. The algorithm maintains a fixed-sized archive of solutions. The archive is trimmed using environmental selection, whereas fitness values are computed by combining the number of population members that a solution dominates and its distance to the k-nearest neighbour.

The recently developed steady-state ESPEA has exhibited excellent results on many popular artificial benchmark problems [3], which is why it was chosen for this study. It mimics the physical phenomenon of electrostatic potential energy by finding a Pareto front approximation that minimizes the overall sum of all pairwise inverse distances between individual solutions in the objective space. For this purpose, the algorithm maintains a variable-sized archive of non-dominated solutions. After the archive has reached its maximum size, a new solution can only replace an existing archive member if the replacement reduces the sum of pairwise inverse distances.

All algorithms were run using a population size of 100 . Each run was terminated after 50,000 function evaluations. Binary crossover and bit flip mutation were applied as genetic operators. A crossover probability of 0.9 and a mutation probability of one by the number of decision variables were chosen. Every algorithm was run 100 times on each respective scenario.

\section{Results and Discussion}

Our analysis is divided into two parts. We first assess how the selected algorithms perform in finding a representative approximation of the Pareto front of the different problem scenarios. The second part concerns itself with how well the multi-objective approach is suited to achieve individual objectives by comparing extreme solutions. The temporal resolution was set to $60 \mathrm{~s}$ in the first and one second in the second part of the analysis.

\subsection{Median Performance Evaluation}

We use the hypervolume [25] and the inverted generational distance (IGD) [26] to evaluate performances, since the two indicators capture both diversity and convergence. Both indicators were computed for every final population of each algorithm and run. Hypervolume measures the space that is covered by the 
Table 5. Hypervolume. Medians and inter-quartile ranges.

\begin{tabular}{lllll}
\hline & ESPEA & NSGA-II & NSGA-III & SPEA2 \\
\hline RW1 & $2.72 E-015.5 E-02$ & $2.42 E-015.3 E-02$ & $1.67 E-015.8 E-02$ & $2.37 E-015.8 E-02$ \\
RW2 & $2.69 E-014.3 E-02$ & $2.42 E-013.4 E-02$ & $1.77 E-015.0 E-02$ & $2.52 E-014.0 E-02$ \\
RW3 & $2.68 E-013.8 E-02$ & $2.37 E-012.8 E-02$ & $1.78 E-015.6 E-02$ & $2.51 E-014.3 E-02$ \\
RS1 & $3.97 E-014.5 E-02$ & $3.98 E-012.5 E-02$ & $2.91 E-011.2 E-01$ & $3.90 E-012.6 E-02$ \\
RS2 & $4.61 E-013.6 \mathrm{E}-02$ & $4.59 E-012.0 E-02$ & $3.53 E-011.5 E-01$ & $4.48 E-014.1 E-02$ \\
RS3 & $3.77 E-013.7 E-02$ & $3.77 E-012.7 E-02$ & $2.79 E-019.6 E-02$ & $3.68 E-013.3 E-02$ \\
CB & $2.45 E-011.8 E-02$ & $2.30 E-011.5 E-02$ & $4.39 E-023.8 E-02$ & $2.04 E-013.1 E-02$ \\
\hline
\end{tabular}

Pareto front approximation with respect to a given reference point, so a larger value is considered to be superior. The IGD indicator is computed by discretizing the Pareto front in a finite set of points and calculating the minimum distance of each said point to the Pareto front approximation. The average of all distances yields the IGD value, which makes smaller values preferable. Since the true Pareto fronts of all problem instances are unknown, reference fronts were created by combining the non-dominated solutions from all individual runs. Objective values were normalized before indicator values were calculated.

An Anderson-Darling test rejected the hypothesis of normal distribution for both indicator values across all scenarios, which is why we report medians and interquartile ranges. Table 5 shows medians and inter-quartile ranges (as sub-scripts) of the hypervolume indicator across all 100 runs. Best and second-best median performances are colored in dark and light gray, respectively. We observe that ESPEA and NSGA-II exhibit the overall largest hypervolumes. SPEA2 is only able to achieve two second-best performances and NSGA-III obtains no best or secondbest performance. Performance differences were checked for significance using a Kruskal-Wallis test in combination with a post-hoc analysis. Significant differences between NSGA-II and ESPEA could be confirmed for scenarios RW2, RW3 and OB. Since the differences for scenario RS1 and RS3, in which NSGA-II outperforms ESPEA, were not found to be significant, we can draw the conclusion that ESPEA achieves the overall best performance with respect to the hypervolume indicator.

A similar pattern can be observed for the IGD indicator, whose results are displayed in Table 6. ESPEA and NSGA-II again nearly achieve all best and second-best performances. ESPEA even outperforms NSGA-II on all scenarios with the exception of the commercial building. Performance differences between both algorithms were found to be significant with the exception of the scenarios of the residential buildings in summer.

The values in Tables 5 and 6 support the conclusion that all algorithms are capable of finding good approximations of the Pareto fronts. This enables users to choose a schedule among multiple balanced options that serves their interests best. However, at the same time, we observe that significant performance differences exist among the algorithms analyzed in this study. Table 7 provides a 
summary of the significant performance differences that are observed in the data. The numbers support our previous analysis that ESPEA is the top performing algorithm closely followed by NSGA-II.

We believe that the performance differences observed can be explained by the algorithms' selection mechanisms. The smart building optimization problem as presented in this study is discrete. Reference point based methods such as NSGAIII, although tailored for high dimensional problems, may struggle to find a diverse approximation of the Pareto front. Points of the Pareto front might be unequally distributed, which could make an association with reference points that span the entire front an unfavorable density estimate. The objective space being discrete might also impair SPEA2's environmental selection mechanism as k-nearest distances are largely affected by the density of points along each objective. Crowding distance based selection mechanisms are known to perform poorly in higher dimensions [27]. NSGA-Il's good results can be explained, however, by very small differences in objectives being less probable to occur. Marginal differences distort the crowding distance metric, as absolute differences to neighboring solutions are multiplied along each dimension. ESPEA's selection mechanism, on the other hand, is not negatively affected by discrete objective spaces, which is reflected by the results.

\subsection{Comparison of Extreme Values}

Increasing the temporal resolution to one second gives us a clear picture of the greatest improvements that can be achieved using optimization techniques. Tables 8 and 9 list the minimum values that were achieved for each objective and algorithm. Best and second-best performances are again colored in dark and light gray. Colorings are omitted if best and second-best performances are tied. Technical wearout is not deeper considered in this analysis, since optimal values are easily achieved and resulting objective vectors are highly dissimilar.

ESPEA and NSGA-II are again the best performing algorithms. At the same time however, the results of NSGA-III and SPEA2 greatly improve. This could be attributed to the higher temporal resolution, which is expected to increase the density of points in the objective space. We can also observe a clear difference

Table 6. IGD. Medians and inter-quartile ranges.

\begin{tabular}{lllll}
\hline & ESPEA & NSGA-II & NSGA-III & SPEA2 \\
\hline RW1 & $2.31 E-032.5 E-03$ & $4.24 E-031.3 E-03$ & $6.18 E-031.8 E-03$ & $4.74 E-038.1 E-04$ \\
RW2 & $2.34 E-032.0 E-03$ & $4.07 E-037.9 E-04$ & $6.10 E-032.4 E-03$ & $4.16 E-038.2 E-04$ \\
RW3 & $2.33 E-032.1 E-03$ & $4.20 E-035.6 E-04$ & $6.13 E-032.3 E-03$ & $4.34 E-039.0 E-04$ \\
RS1 & $3.38 E-031.1 E-03$ & $3.51 E-036.8 E-04$ & $5.93 E-032.8 E-03$ & $4.34 E-038.4 E-04$ \\
RS2 & $3.40 E-038.3 E-04$ & $3.44 E-035.2 E-04$ & $5.53 E-032.7 E-03$ & $4.06 E-036.8 E-04$ \\
RS3 & $3.44 E-038.6 E-04$ & $3.72 E-035.0 E-04$ & $6.43 E-033.4 E-03$ & $4.40 E-039.3 E-04$ \\
CB & $2.03 E-032.5 E-04$ & $1.81 E-031.1 E-04$ & $4.57 E-039.9 E-04$ & $1.96 E-031.6 E-04$ \\
\hline
\end{tabular}


Table 7. Summary statistics for the hypervolume and IGD indicators. The number in each column states how many times the corresponding algorithm outperforms other algorithms with confidence across all scenarios and indicators at a five percent level.

\begin{tabular}{l|l|l|l|l}
\hline & ESPEA & NSGA-II & NSGA-III & SPEA2 \\
\hline Performance & 28 & 24 & 0 & 14 \\
\hline
\end{tabular}

Table 8. Extreme solutions of RW and RS scenarios. Each cell entry is composed of the tuple costs, emissions, thermal discomfort, and wearout.

\begin{tabular}{cllll}
\hline \multicolumn{3}{c}{ Costs } & CO2 emissions & Thermal discomfort \\
\hline RW1 ESPEA & $(1803,44165,1.94,3)$ & $(1833,44126,1.94,3)$ & $(2039,49935,0.0,3)$ \\
NSGA-II & $(1818,44548,1.85,4)$ & $(1838,44418,1.91,4)$ & $(2044,49982,0.0,4)$ \\
NSGA-III & $(1835,44560,1.88,5)$ & $(1837,44535,1.88,5)$ & $(2060,50319,0.0,4)$ \\
SPEA2 & $(1824,44757,1.86,4)$ & $(1845,44651,1.91,4)$ & $(2022,50061,0.0,4)$ \\
\hline RW2 ESPEA & $(1800,44062,1.94,4)$ & $(1825,44045,1.94,4)$ & $(2091,52415,0.0,3)$ \\
& NSGA-II & $(1805,44231,1.92,4)$ & $(1819,44207,1.93,4)$ & $(2020,49979,0.0,4)$ \\
& NSGA-III & $(1818,44529,1.91,5)$ & $(1824,44525,1.91,5)$ & $(2030,50167,0.0,5)$ \\
& SPEA2 & $(1815,44494,1.87,4)$ & $(1833,44429,1.89,5)$ & $(2024,50095,0.0,4)$ \\
\hline RW3 ESPEA & $(1809,44392,1.90,3)$ & $(1822,44378,1.90,3)$ & $(2015,49912,0.0,3)$ \\
& NSGA-II & $(1797,44103,1.93,4)$ & $(1818,44086,1.93,4)$ & $(2012,49841,0.0,3)$ \\
& NSGA-III & $(1817,44624,1.86,4)$ & $(1817,44624,1.86,4)$ & $(2046,50645,0.0,4)$ \\
& SPEA2 & $(1801,44174,1.93,4)$ & $(1835,44032,1.94,4)$ & $(2023,50095,0.0,4)$ \\
\hline RS1 & ESPEA & $(-119,6224,3.38,3)$ & $(-119,6224,3.38,3)$ & $(374,14650,0.05,6)$ \\
& NSGA-II & $(-137,5713,3.52,3)$ & $(-137,5713,3.52,3)$ & $(363,14106,0.06,6)$ \\
& NSGA-III & $(-104,6551,3.49,4)$ & $(-104,6551,3.49,4)$ & $(310,13935,0.38,7)$ \\
& SPEA2 & $(-119,6195,3.57,3)$ & $(-119,6195,3.57,3)$ & $(364,14182,0.06,5)$ \\
\hline RS2 & ESPEA & $(-183,4479,4.03,4)$ & $(-183,4479,4.03,4)$ & $(330,13558,0.08,6)$ \\
\hline & NSGA-II & $(-122,6125,3.50,3)$ & $(-122,6125,3.50,3)$ & $(412,15282,0.10,8)$ \\
& NSGA-III & $(-150,5363,3.82,4)$ & $(-150,5363,3.82,4)$ & $(334,13733,0.09,6)$ \\
\hline SPEA2 & $(-113,6354,3.54,3)$ & $(-113,6354,3.54,3)$ & $(356,14148,0.11,5)$ \\
\hline RS3 & ESPEA & $(-110,6445,3.30,3)$ & $(-110,6445,3.30,3)$ & $(318,13706,0.11,7)$ \\
& NSGA-II & $(-114,6373,3.27,3)$ & $(-114,6372,3.41,3)$ & $(333,13868,0.05,6)$ \\
\hline NSGA-III & $(-122,6095,3.58,3)$ & $(-122,6095,3.58,3)$ & $(306,13571,0.24,6)$ \\
& SPEA2 & $(-118,6262,3.47,3)$ & $(-118,6262,3.47,3)$ & $(298,13273,0.21,6)$ \\
\hline
\end{tabular}

between the summer and winter scenarios. The PV system makes the residential household a net power supplier, as it provides more power than the household consumes. This circumstance leads the minimum cost and minimum emission solutions to coincide in summer, which demonstrates that private PV systems may reconcile those two conflicting objectives.

The comparison of summer and winter scenarios reveals that the additional degree of freedom provided by interruptible and load flexible appliances leads 
Table 9. Extreme solutions of scenario CB. Each cell entry is composed of the tuple costs, emissions, thermal discomfort, visual discomfort, and wearout.

\begin{tabular}{lll}
\hline \multicolumn{2}{c}{ Costs } & CO2 emissions \\
\hline ESPEA & $(4800,127197,3.45,99.8,3)$ & $(5235,99613,3.36,99.0,6)$ \\
NSGA-II & $(4848,128521,3.40,98.5,2)$ & $(5213,112008,3.32,98.5,5)$ \\
NSGA-III & $(5288,140215,3.14,35.7,4)$ & $(5364,138639,3.14,20.0,4)$ \\
SPEA2 & $(4880,128856,3.41,96.1,4)$ & $(5371,116884,3.30,97.1,8)$ \\
\hline & Thermal discomfort & Visual discomfort \\
\hline ESPEA & $(7095,189775,0.3,99.0,3)$ & $(6338,160802,1.78,0.00,2)$ \\
NSGA-II & $(7546,192995,0.3,0.00,3)$ & $(7508,200388,0.31,0.00,3)$ \\
NSGA-III & $(7475,199820,0.3,6.60,4)$ & $(7475,199820,0.30,6.60,4)$ \\
SPEA2 & $(7389,196964,0.3,28.8,4)$ & $(5473,144760,2.79,27.5,4)$ \\
\hline
\end{tabular}

in most cases to an improvement in objective values. Consumption flexibility is therefore suitable to reduce costs and emissions, while raising comfort at the same time. Such an improvement, however, cannot be confirmed for all scenarios. We believe that deteriorating objective values that have been observed in some cases are caused by the additional complexity introduced by increasing the search space. We believe that focusing the search from the beginning on identifying tradeoff solutions that balance objective values, for example by applying methods from [28], might remedy this situation.

\section{Conclusion and Outlook}

We have presented a new approach of formulating a smart building multiobjective optimization problem. Our formulation introduces device encodings that allow for the generic modeling of interruptible and load-flexible appliances and make constraint satisfaction easier to achieve. Our computational study has revealed that current multi-objective evolutionary algorithms are able to compute representative approximations of the Pareto front and approximate extreme solutions alike. Among the four tested state-of-the-art algorithms, ESPEA and NSGA-II delivered the overall best results.

Future research may focus on developing specialized genetic operators and repair mechanisms tailored to our device encodings for improving MO-EA performances. Additionally, further focus can be placed on identifying tradeoff solutions that balance objective values.

An implementation of a multi-objective evolutionary algorithm based on the results in this paper will be transferred to the building energy management system in our laboratory environments and tested with real test users. This will help verifying the results obtained in the present analysis. 


\section{References}

1. Gottwalt, S., Ketter, W., Block, C., Collins, J., Weinhardt, C.: Demand side management - a simulation of household behavior under variable prices. Energy Policy 39(12), 8163-8174 (2011)

2. Palensky, $P$., Dietrich, D.: Demand side management: demand response, intelligent energy systems, and smart loads. IEEE Trans. Industr. Inf. 7(3), 381-388 (2011)

3. Braun, M.A., Shukla, P.K., Schmeck, H.: Obtaining optimal pareto front approximations using scalarized preference information. In: Proceedings of the 2015 Annual Conference on Genetic and Evolutionary Computation, GECCO 2015, pp. 631-638. ACM, New York (2015)

4. Deb, K., Pratap, A., Agarwal, S., Meyarivan, T.: A fast and elitist multiobjective genetic algorithm: NSGA-II. IEEE Trans. Evol. Comput. 6(2), 182-197 (2002)

5. Deb, K., Jain, H.: An evolutionary many-objective optimization algorithm using reference-point-based nondominated sorting approach, part I: solving problems with box constraints. IEEE Trans. Evol. Comput. 18(4), 577-601 (2014)

6. Zitzler, E., Laumanns, M., Thiele, L.: SPEA2: improving the strength Pareto evolutionary algorithm. Technical report 103, Computer Engineering and Networks Laboratory (TIK), Swiss Federal Institute of Technology (ETH), Zurich, Switzerland (2001)

7. Kok, J.K., Warmer, C.J., Kamphuis, I.G.: PowerMatcher: multiagent control in the electricity infrastructure. In: Proceedings of the Fourth International Joint Conference on Autonomous Agents and Multiagent Systems, AAMAS 2005, pp. 7582. ACM, New York (2005)

8. Ha, D.L., Joumaa, H., Ploix, S., Jacomino, M.: An optimal approach for electrical management problem in dwellings. Energy Build. 45, 1-14 (2012)

9. Nestle, D., Bendel, C., Ringelstein, J.: Bidirectional energy management interface (BEMI)-integration of the low voltage level into grid communication and control. In: 19th International Conference on Electricity Distribution, pp. 21-24, Vienna (2007)

10. Soares, A., Gomes, A., Antunes, C.H.: Integrated management of residential energy resources. In: EPJ Web of Conferences, vol. 33 (2012)

11. Mauser, I., M"uller, J., Allerding, F., Schmeck, H.: Adaptive building energy management with multiple commodities and flexible evolutionary optimization. Renewable Energy 87(Part 2), 911-921 (2016)

12. Ahmadi, P., Rosen, M.A., Dincer, I.: Multi-objective exergy-based optimization of a polygeneration energy system using an evolutionary algorithm. Energy 46(1), 2131 (2012)

13. Allerding, F., Premm, M., Shukla, P.K., Schmeck, H.: Electrical load management in smart homes using evolutionary algorithms. In: Hao, J.-K., Middendorf, M. (eds.) EvoCOP 2012. LNCS, vol. 7245, pp. 99-110. Springer, Heidelberg (2012)

14. Soares, A., Antunes, C.H., Oliveira, C., Gomes, A.: A multi-objective genetic approach to domestic load scheduling in an energy management system. Energy 77 , 144-152 (2014)

15. Zhao, Z., Lee, W.C., Shin, Y., Song, K.B.: An optimal power scheduling method for demand response in home energy management system. IEEE Trans. Smart Grid 4(3), 1391-1400 (2013)

16. Anvari-Moghaddam, A., Seifi, A., Niknam, T., Pahlavani, M.R.A.: Multi-objective operation management of a renewable MG (micro-grid) with back-up microturbine/fuel cell/battery hybrid power source. Energy 36(11), 6490-6507 (2011) 
17. Pedrasa, M., Spooner, T., MacGill, I.: Coordinated scheduling of residential distributed energy resources to optimize smart home energy services. IEEE Trans. Smart Grid 1(2), 134-143 (2010)

18. De Oliveira, G., Jacomino, M., Ploix, S.: Optimal power control for smart homes. In: IFAC World Congr'ess, Milan, Italy, pp. 9579-9586 (2011)

19. Salinas, S., Li, M., Li, P.: Multi-objective optimal energy consumption scheduling in smart grids. IEEE Trans. Smart Grid 4(1), 341-348 (2013)

20. Mauser, I., Dorscheid, M., Allerding, F., Schmeck, H.: Encodings for Evolutionary Algorithms in smart buildings with energy management systems. In: IEEE Congress on Evolutionary Computation (CEC), pp. 2361-2366. IEEE (2014)

21. Durillo, J.J., Nebro, A.J.: The jMetal framework for multi-objective optimization: design and architecture. Adv. Eng. Softw. 42(10), 760-771 (2011)

22. Dallinger, D.: The contribution of vehicle-to-grid to balance fluctuating generation: comparing different battery ageing approaches. Technical report, Working Paper Sustainability and Innovation (2013)

23. Reinhart, C.F., Herkel, S.: The simulation of annual daylight illuminance distributions a state-of-the-art comparison of six RADIANCE-based methods. Energy Build. 32(2), 167-187 (2000)

24. Graditi, G., Di Silvestre, M., Gallea, R., Sanseverino, E.R.: Heuristic-based shiftable loads optimal management in smart micro-grids. IEEE Trans. Industr. Inf. 11(1), 271-280 (2015)

25. Zitzler, E., Thiele, L.: Multiobjective evolutionary algorithms: a comparative case study and the strength Pareto approach. IEEE Trans. Evol. Comput. 3(4), 257-271 (1999)

26. Van Veldhuizen, D.A., Lamont, G.B.: Evolutionary computation and convergence to a Pareto front. In: Late Breaking Papers at the Genetic Programming 1998 Conference, pp. 221-228. Citeseer (1998)

27. Kukkonen, S., Deb, K.: Improved pruning of non-dominated solutions based on crowding distance for bi-objective optimization problems. In: Proceedings of the World Congress on Computational Intelligence, Vancouver, Canada, pp. 11791186. IEEE Press (2006)

28. Shukla, P.K., Braun, M.A., Schmeck, H.: Theory and algorithms for finding knees. In: Purshouse, R.C., Fleming, P.J., Fonseca, C.M., Greco, S., Shaw, J. (eds.) EMO 2013. LNCS, vol. 7811, pp. 156-170. Springer, Heidelberg (2013) 